\title{
SOME LIMIT THEOREMS RELATED TO MULTI-DIMENSIONAL DIFFUSIONS IN A RANDOM ENVIRONMENT
}

\author{
DAEHONG KIM
}

\begin{abstract}
In this paper, we consider a multi-dimensional diffusion process in a self-similar random environment and prove a limit theorem for the shape of the full trajectory of the diffusion by using the localization phenomenon.
\end{abstract}

\section{Introduction}

Let $\mathcal{W}$ be the space of continuous functions on $\mathbf{R}^{n}$ vanishing at the origin and let $Q$ be a probability measure on it. We call an element of $\mathcal{W}$ an environment. For given an environment $w$, let $\mathbf{P}_{x}^{w}$ be the probability measure on $\Omega$, the canonical path space of real-valued continuous functions from $[0, \infty)$ to $\mathbf{R}^{n}$, such that $\left\{X(t), \mathbf{P}_{x}^{w}, x \in \mathbf{R}^{n}\right\}$ is a diffusion process with generator

$$
\mathcal{L}^{w}=\frac{1}{2} e^{w} \sum_{i=1}^{n} \frac{\partial}{\partial x_{i}}\left(e^{-w} \frac{\partial}{\partial x_{i}}\right)
$$

It is well known that such a process $X(t)$ can be constructed from a Brownian motion by a drift transformation ([2]).

Let $\mathcal{P}_{x}$ be the probability measure on $\mathcal{W} \times \Omega$ defined by $\mathcal{P}_{x}(d w d \omega)=$ $Q(d w) \mathbf{P}_{x}^{w}(d \omega)$. Then the diffusion $X(t)$ can be regarded as a stochastic process defined on the probability space $\left(\mathcal{W} \times \Omega, \mathcal{P}_{x}\right)$ and it is called as a diffusion process in a random environment. This process has a close connection with the model of Sinai's random walk and exhibits some interesting features ([1], [5], [8], [9], [10]). Among those, Brox [1] showed when $n=1$ and $w(x)$ is a Brownian environment that there exists a nontrivial measurable function $b_{1}: \mathcal{W} \mapsto \mathbf{R}$ such that for any $\varepsilon>0$,

$$
\mathcal{P}_{x}\left(\left|\alpha^{-2} X\left(e^{\alpha}\right)-b_{1}(w)\right|>\varepsilon\right) \longrightarrow 0 \quad \text { as } \alpha \rightarrow \infty
$$

Received May 11, 2009; Revised October 13, 2009.

2010 Mathematics Subject Classification. Primary 60K37, 31C25, 60J60.

Key words and phrases. Dirichlet forms, random environment, set valued diffusion processes, subdiffusivity.

This research was partially supported by Grant-in-Aid for Scientific Research No. 17740062. 
which is the so-called subdiffusivity. This result was a consequence of a localization phenomenon, the diffusion is trapped in some valleys of its potential $w$, and was extended to a large class of random environments ([5]). However, all their methods are heavily rely on the one dimensional situation.

The first analogue result of (2) in higher dimensional cases was obtained by Mathieu [8]. The novelty of his work was the introduction of some analytic approaches such as asymptotics of the first non-zero eigenvalue of the generator (1) with a small noise and the related Dirichlet form theory. Motivated by this work, Tanaka [13] also proved that $\left\{X(t), \mathcal{P}_{x}, x \in \mathbf{R}^{n}\right\}$ is to be recurrent for all dimensions.

We are interested in the long time asymptotics for the shape of the full trajectory of the multi-dimensional diffusion $X(t)$ in a random environment. More precisely, let us consider the random set of trajectory of the diffusion $X(t)$ up to time $t$ :

$$
\mathcal{X}(t):=\overline{\{X(s): 0 \leq s \leq t\}}
$$

where $\bar{A}$ stands for the closure of a set $A$. We may call the process $\mathcal{X}(t)$ on the probability space $\left(\mathcal{W} \times \Omega, \mathcal{P}_{x}, x \in \mathbf{R}^{n}\right)$ a set valued diffusion process in a random environment. Our purpose in this article is to extend the result (2) of Brox to the case of set valued diffusion $\mathcal{X}(t)$.

To state our main theorem precisely, let $\mathcal{K}$ be a family of non-empty compact sets of $\mathbf{R}^{n}$ and $D_{r}(w)$ the connected component containing the origin of the sub-level domain $\left\{x \in \mathbf{R}^{n}: w(x)<r\right\}$. Let $d_{H}$ be the Hausdorff distance on $\mathcal{K}$, that is, for $K_{1}, K_{2} \in \mathcal{K}$

$$
d_{H}\left(K_{1}, K_{2}\right)=\inf \left\{\varepsilon>0: U_{\varepsilon}\left(K_{1}\right) \supset K_{2}, U_{\varepsilon}\left(K_{2}\right) \supset K_{1}\right\},
$$

where $U_{\varepsilon}(K)$ denotes the $\varepsilon$-neighborhood of $K$. Our result is based on some assumptions for the random characteristics of the environment. The main result of this article is stated as follows:

Theorem 1.1. Let $f$ be a probability density on $\mathbf{R}^{n}$ with compact support. Suppose that

(A.1) For any $\alpha>0$ and any fixed $\lambda>0$, the environment

$$
\left\{w_{\alpha, \lambda}(x):=\alpha^{-1} w\left(\alpha^{\lambda} x\right), x \in \mathbf{R}^{n}\right\}
$$

has the same law as $\left\{w(x), x \in \mathbf{R}^{n}\right\}$ under $Q$, namely $(\mathcal{W}, Q)$ is a $\lambda^{-1}$-self-similar random environment.

(A.2) For $Q$-a.s. $D_{r}(w)$ is bounded for any $r>0$ and for $Q$-a.s. $w(x) \geq 0$ for all $x \in \mathbf{R}^{n}$.

Then for any $r>0, \varepsilon>0$ and any fixed $\lambda>0$,

$$
\mathcal{P}_{f}\left(d_{H}\left(\alpha^{-\lambda} \mathcal{X}\left(e^{\alpha r}\right), \overline{D_{r}\left(w_{\alpha, \lambda}\right)}\right)>\varepsilon\right) \longrightarrow 0 \quad \text { as } \alpha \rightarrow \infty .
$$

That is, the law of the random set $\alpha^{-\lambda} \mathcal{X}\left(e^{\alpha r}\right)$ under $\mathcal{P}_{f}$ converges to the law of the compact set $\overline{D_{r}(w)}$ under $Q$ as $\alpha \rightarrow \infty$. 
The natural and typical examples of the random environment satisfying the assumptions (A.1) and (A.2) can be provided by $\mathcal{W}=\{w: w(x)=|B(x)|, x \in$ $\left.\mathbf{R}^{n}\right\}$, where $B(x)$ is a Lévy's Brownian motion with a $n$-dimensional time (cf. [8]) and $\mathcal{W}=\left\{w: w(x)=\sum_{i=1}^{n}\left|B\left(x_{i}\right)\right|, x=\left(x_{1}, x_{2}, \ldots, x_{n}\right) \in \mathbf{R}^{n}\right\}$, where $\left\{B_{i}(x)\right\}_{1 \leq i \leq n}$ is a family of one dimensional Brownian motions which are mutually independent (cf. [12]).

Our theorem tells us that the shape of the $\mathcal{K}$-valued stochastic process $r^{\lambda} \mathcal{X}(t)$ converges very slowly to that of the connected component containing the origin of $\left\{x \in \mathbf{R}^{n}: w(x)<r\right\}$ by the effect of environment.

To prove our theorem, we address in Section 2 some notations and facts on a one parameter family of diffusion processes and their associated Dirichlet forms (for the general theory of Dirichlet forms, we refer readers to [2]). In Section 3 , we obtain some asymptotic results on the first exit times of parameterized diffusions originally due to Mathieu [8] with some modifications (cf. [6], [7]). The proof of the main theorem will be given in Section 4. A key point of the proof is to use a modified environment to show that the parameterized diffusions enter any ball before leaving the domain $D_{r}(w)$.

\section{Preliminaries on parameterized diffusions}

For a given $w \in \mathcal{W}$, consider a symmetric closable form on $L^{2}\left(\mathbf{R}^{n} ; e^{-\alpha w} d x\right)$ (energy form) parameterized by $\alpha>0$ :

$$
\mathcal{E}^{\alpha w}(u, v)=\frac{1}{2} \int_{\mathbf{R}^{n}} \nabla u(x) \cdot \nabla v(x) e^{-\alpha w(x)} d x, \quad u, v \in C_{0}^{1}\left(\mathbf{R}^{n}\right),
$$

where $C_{0}^{1}\left(\mathbf{R}^{n}\right)$ is the space of continuously differentiable functions with compact support in $\mathbf{R}^{n}$. Then $\left(\mathcal{E}^{\alpha w}, H^{1}\left(\mathbf{R}^{n}\right)\right)$ becomes a regular (local) Dirichlet form defined by the smallest closed extension of (3) (see [2]). Here $H^{1}\left(\mathbf{R}^{n}\right)=\{u \in$ $\left.L^{2}\left(\mathbf{R}^{n}\right): \partial_{i} u \in L^{2}\left(\mathbf{R}^{n}\right), 1 \leq i \leq n\right\}$. By a general theory of Dirichlet forms, there exists a diffusion process associated with $\left(\mathcal{E}^{\alpha w}, H^{1}\left(\mathbf{R}^{n}\right)\right)$ and we denote it by $\left\{X(t), \mathbf{P}_{x}^{\alpha w}, x \in \mathbf{R}^{n}\right\}$. Let $f$ be a probability density function on $\mathbf{R}^{n}$ and set

$$
\mathbf{P}_{f}(\cdot):=\int_{\mathbf{R}^{n}} \mathbf{P}_{x}(\cdot) f(x) d x
$$

for a probability distribution $\mathbf{P}_{x}$. Then $\left\{X(t), \mathbf{P}_{f}^{\alpha w}\right\}$ can be regarded as the diffusion process with an initial distribution $f$ of the generator (1) replaced $w$ by $\alpha w$.

For a fixed $\lambda>0$, we simply write as

$$
w_{\alpha}(x):=\alpha^{-1} w\left(\alpha^{\lambda} x\right), \quad f_{\alpha}(x):=\alpha^{\lambda n} f\left(\alpha^{\lambda} x\right), x \in \mathbf{R}^{n} .
$$

Let $\left\{R_{\beta}^{w}\right\}_{\beta>0}$ be the resolvent of $\left\{X(t), \mathbf{P}_{f}^{w}\right\}$. Then for any $\varphi \in L^{2}\left(\mathbf{R}^{n} ; e^{-w} d x\right)$ and $v \in H^{1}\left(\mathbf{R}^{n}\right)$,

$$
\mathcal{E}_{\beta}^{w}\left(R_{\beta}^{w} \varphi, v\right)=\int_{\mathbf{R}^{n}} \varphi(x) v(x) e^{-w(x)} d x
$$


where $\mathcal{E}_{\beta}^{w}(\phi, \psi):=\mathcal{E}^{w}(\phi, \psi)+\beta \int_{\mathbf{R}^{n}} \phi(x) \psi(x) e^{-w(x)} d x$ for $\phi, \psi \in H^{1}\left(\mathbf{R}^{n}\right)$. By the change of variable with $x=\alpha^{\lambda} y$, the equation (5) yields that

$$
\begin{aligned}
\mathcal{E}_{\alpha^{2 \lambda} \beta}^{\alpha w_{\alpha}}\left(R_{\beta}^{w} \varphi\left(\alpha^{\lambda} \cdot\right), v\left(\alpha^{\lambda} \cdot\right)\right) & =\int_{\mathbf{R}^{n}} \alpha^{2 \lambda} \varphi\left(\alpha^{\lambda} y\right) v\left(\alpha^{\lambda} y\right) e^{-\alpha w_{\alpha}(y)} d y \\
& =\mathcal{E}_{\alpha^{2 \lambda} \beta}^{\alpha w_{\alpha}}\left(R_{\alpha^{2 \lambda} \beta}^{\alpha w_{\alpha}}\left(\alpha^{2 \lambda} \varphi\right)\left(\alpha^{\lambda} \cdot\right), v\left(\alpha^{\lambda} \cdot\right)\right) .
\end{aligned}
$$

So the fact that $\left\{\alpha^{2 \lambda} R_{\alpha^{2 \lambda} \beta}^{\alpha w_{\alpha}}\right\}_{\beta>0}$ is the resolvent of $\left\{\alpha^{\lambda} X\left(\alpha^{-2 \lambda} t\right), \mathbf{P}_{f_{\alpha}}^{\alpha w_{\alpha}}\right\}$ implies the following lemma.

Lemma 2.1. For $w \in \mathcal{W}$ and $\alpha>0$,

$$
\left\{\alpha^{-\lambda} X(t), \mathbf{P}_{f}^{w}\right\} \stackrel{d}{=}\left\{X\left(\alpha^{-2 \lambda} t\right), \mathbf{P}_{f_{\alpha}}^{\alpha w_{\alpha}}\right\}
$$

where $\stackrel{d}{=}$ means the equality in distribution.

For any $r>0$, let $r(\alpha):=r-(2 \lambda / \alpha) \log \alpha$. Clearly $r(\alpha) \rightarrow r$ as $\alpha \rightarrow \infty$. Then (6) can be rewritten as

$$
\left\{\alpha^{-\lambda} X\left(e^{\alpha r}\right), \mathbf{P}_{f}^{w}\right\} \stackrel{d}{=}\left\{X\left(e^{\alpha r(\alpha)}\right), \mathbf{P}_{f_{\alpha}}^{\alpha w_{\alpha}}\right\}
$$

by taking $t=e^{\alpha r}$.

Remark 2.2. Under the assumption (A.1), $\mathbf{P}^{\alpha w_{\alpha}}$ and $\mathbf{P}^{\alpha w}$ are same for $Q$ a.s.. Therefore we see from (7) that a subdiffusivity problem of the diffusion $X(t)$ is reformulated as an $\alpha$-asymptotic problem of the parameterized diffusion $\left\{X\left(e^{\alpha r(\alpha)}\right), \mathcal{P}_{f_{\alpha}}^{\alpha}\right\}$. Here $\mathcal{P}_{x}^{\alpha}(d w d \omega):=Q(d w) \mathbf{P}_{x}^{\alpha w}(d \omega)$.

Let $D$ be an arbitrary bounded domain in $\mathbf{R}^{n}$. We introduce the so-called 3-depths of $D$ relative to an environment (cf. [4], [8]): the depths of $D$,

$$
d=d(D, w)=\inf _{\partial D} w-\inf _{D} w, \quad d^{\prime}=d^{\prime}(D, w)=\sup _{D} w-\inf _{D} w
$$

and the critical depth (or elevation) of $D$,

$$
c=c(D, w)=\sup _{x, y \in D}\left\{\inf _{\phi} \sup _{t \in[0,1]} w(\phi(t))-w(x)-w(y)\right\}+\inf _{D} w
$$

where $\phi$ is a continuous path from $[0,1]$ to $D$ such that $\phi(0)=x, \phi(1)=y$. Note that the quantities $d^{\prime}, d$ and $c$ depend only on the landscape of $w$ and do not depend on any boundary conditions (such as smoothness) of $\partial D$. Moreover, it is easy to check that if $D \equiv D_{r}(w)(r>0)$, then $d^{\prime}=d$ and $d>c$.

Let $H^{1}(D)=\left\{u \in L^{2}(D): \partial_{i} u \in L^{2}(D), 1 \leq i \leq n\right\}$ and denotes $H_{0}^{1}(D)$ by the closure of $C_{0}^{1}(D)$ in $H^{1}(D)$. It is well known that the absorbing process of the diffusion $\left\{X(t), \mathbf{P}_{x}^{\alpha w}, x \in \mathbf{R}^{n}\right\}$ on $D$ is by definition the diffusion process $\left\{X(t), \mathbf{P}_{x}^{\alpha w, D}, x \in D\right\}$ whose regular Dirichlet form on $L^{2}\left(D ; e^{-\alpha w} d x\right)$ is coincided with $\left(\mathcal{E}^{\alpha w, D}, H_{0}^{1}(D)\right)$, where

$$
\mathcal{E}^{\alpha w, D}(u, v)=\frac{1}{2} \int_{D} \nabla u(x) \cdot \nabla v(x) e^{-\alpha w(x)} d x
$$


([2]). On the other hand, by defining $H_{*}^{1}(D):=\left(H_{0}^{1}(D)+\right.$ constant $)$ as a new Sobolev space, $\left(\mathcal{E}^{\alpha w, D}, H_{*}^{1}(D)\right)$ becomes a regular Dirichlet form on $L^{2}\left(D^{*} ; e^{-\alpha w} d x\right)$ as well $([6])$. In fact, this form is the smallest one in the family of Dirichlet forms $\mathcal{M}=\left\{\left(\mathcal{E}^{\alpha w, D}, \mathcal{F}^{D}\right): H_{0}^{1}(D) \subset \mathcal{F}^{D} \subseteq H^{1}(D), 1 \in\right.$ $\left.\mathcal{F}^{D}\right\}$. Here $D^{*}$ denotes the one-point compactification of $D$. Let us denote by $\left\{X(t), \mathbf{P}_{x}^{\alpha w, *}, x \in D^{*}\right\}$ the diffusion process associated with $\left(\mathcal{E}^{\alpha w, D}, H_{*}^{1}(D)\right)$. This process is irreducible and recurrent on $D^{*}$. More general profound properties on this kind of process and its associated Dirichlet form can be found in $[3]$.

For a notational convenience, we set

$$
u(\alpha) \succ a \text { if } \liminf _{\alpha \rightarrow \infty} \frac{1}{\alpha} \log u(\alpha) \geq a, \quad u(\alpha) \prec a \text { if } \limsup _{\alpha \rightarrow \infty} \frac{1}{\alpha} \log u(\alpha) \leq a .
$$

Let $m_{\alpha}(d x)$ be the normalized underlying measure defined by

$$
m_{\alpha}(d x)=I_{D} Z_{\alpha}^{-1} e^{-\alpha w(x)} d x, \quad Z_{\alpha}=\int_{D} e^{-\alpha w(x)} d x .
$$

Note that $(1 / \alpha) \log Z_{\alpha} \rightarrow-\inf _{D} w$ as $\alpha \rightarrow \infty$. Let $\gamma(\alpha)$ be the first non-zero eigenvalue of $\left(\mathcal{E}^{\alpha w, D}, H_{*}^{1}(D)\right)$, the so-called spectral gap, defined by

$$
\gamma(\alpha)=\inf _{u \in H_{*}^{1}(D)} \frac{\mathcal{E}^{\alpha w, D}(u, u)}{\int_{D}\left(u-\int_{D} u d m_{\alpha}\right)^{2} e^{-\alpha w} d x} .
$$

The following asymptotic lower bound of $\gamma(\alpha)$ for an arbitrary bounded domain $D$ of $\mathbf{R}^{n}$ was obtained by [6].

Lemma 2.3. $\gamma(\alpha) \succ-c$.

\section{Lemmas on exit times}

Let $\tau_{D}^{\alpha w}$ be the first exit time of the diffusion $X(t)$ out of $D$, that is, $\tau_{D}^{\alpha w}:=$ $\inf \{t>0: X(t) \notin D\}$. For any $\beta>0$, set $h_{\beta}^{\alpha w}(x):=\mathbf{E}_{x}^{\alpha w, *}\left(\exp \left(-\beta \tau_{D}^{\alpha w}\right)\right)$ the Laplace transform of $\tau_{D}^{\alpha w}$, where $\mathbf{E}_{x}^{\alpha w, *}$ denotes the expectation relative to $\left\{X(t), \mathbf{P}_{x}^{\alpha w, *}, x \in D^{*}\right\}$.

In this section, we consider the asymptotics in $\alpha$ of the distribution of $\tau_{D}^{\alpha w}$ studied in [6], [8] under some modifications. We note that no additional condition is imposed (smoothness as like in [8]) on a domain $D \subset \mathbf{R}^{n}$, and it makes no difference to replace the diffusion $\left\{X(t), \mathbf{P}_{x}^{\alpha w}, x \in \mathbf{R}^{n}\right\}$ by $\left\{X(t), \mathbf{P}_{x}^{\alpha w, *}, x \in D^{*}\right\}$ (or $\left.\left\{X(t), \mathbf{P}_{x}^{\alpha w, D}, x \in D\right\}\right)$ as far as the exit time distribution from $D$ is concerned.

Lemma 3.1. Let $\theta(\alpha)$ be the function such that $\int_{D} h_{\theta(\alpha)}^{\alpha w} d m_{\alpha}=1 / 2$.

(i) $-d^{\prime} \prec \theta(\alpha) \prec-d$.

(ii) Assume $d>c$. Then for any $k>0, \int_{D} h_{k \theta(\alpha)}^{\alpha w} d m_{\alpha} \rightarrow 1 /(1+k)$ as $\alpha \rightarrow \infty$. In particular, set $D \equiv D_{r}(w)(r>0)$. Then for any open ball 
$B$ such that $\bar{B} \subset D$,

(8)

$$
\left\|h_{k \theta(\alpha)}^{\alpha w}-\frac{1}{1+k}\right\|_{L^{2}(B)} \longrightarrow 0
$$

as $\alpha \rightarrow \infty$.

Proof. We may assume that $d>0$ (otherwise the present lemma is trivial). Note that it follows from the proof of Theorem 3.1 in [6] that for any $\varepsilon>0$,

$$
\int_{D} h_{e^{-\alpha(d-\varepsilon)}}^{\alpha w} d m_{\alpha} \longrightarrow 0 \quad \text { as } \alpha \rightarrow \infty \text {. }
$$

On the other hand, for the absorbing diffusion process $\left\{X(t), \mathbf{P}_{x}^{\alpha w, D}, x \in D\right\}$, put $u_{\alpha}^{D}(t, x)=\mathbf{P}_{x}^{\alpha w, D}\left(t<\tau_{D}^{\alpha w}\right)$. Then by definition,

$$
\begin{aligned}
\mathcal{E}^{\alpha w, D}\left(u_{\alpha}^{D}(t, \cdot), u_{\alpha}^{D}(t, \cdot)\right) & =\lim _{s \rightarrow 0}\left(\frac{u_{\alpha}^{D}(t, \cdot)-u_{\alpha}^{D}(s+t, \cdot)}{s}, u_{\alpha}^{D}(t, \cdot)\right)_{e^{-\alpha w} d x} \\
& =-\frac{1}{2} \int_{D} \frac{\partial}{\partial t}\left|u_{\alpha}^{D}(t, x)\right|^{2} e^{-\alpha w(x)} d x \\
& =-\frac{1}{2} \frac{d}{d t} H_{\alpha}^{D}(t),
\end{aligned}
$$

where $(\cdot, \cdot)_{\mu}$ denotes the inner product on $L^{2}(D ; \mu)$ and

$$
H_{\alpha}^{D}(t)=\int_{D}\left|u_{\alpha}^{D}(t, x)\right|^{2} e^{-\alpha w(x)} d x
$$

Let denote $\left(\frac{1}{2} \mathbf{D}, H_{0}^{1}(D)\right)$ by the Dirichlet form $\left(\mathcal{E}^{\alpha w, D}, H_{0}^{1}(D)\right)$ when $\alpha=0$. By the boundedness of $D$, it is transient (Example 1.5.3 in [2]) and thus for any $u \in H_{0}^{1}(D)$, it holds that

$$
\int_{D} u(x)^{2} d x \leq 2\left\|R^{D} 1\right\|_{\infty} \mathbf{D}(u, u)
$$

where $R^{D}$ is the Green operator of $\left(\frac{1}{2} \mathbf{D}, H_{0}^{1}(D)\right)([11])$. Using this, we have

$$
H_{\alpha}^{D}(t) \leq 2\left\|R^{D} 1\right\|_{\infty} e^{\alpha d^{\prime}} \mathcal{E}^{\alpha w, D}\left(u_{\alpha}^{D}(t, \cdot), u_{\alpha}^{D}(t, \cdot)\right) .
$$

By combining (10) and (11),

$$
e^{-\alpha d^{\prime}}\left\|R^{D} 1\right\|_{\infty}^{-1} \leq-\frac{d}{d t} \log H_{\alpha}^{D}(t)
$$

that implies

$$
H_{\alpha}^{D}(t) \leq H_{\alpha}^{D}(0) \exp \left(-\left\|R^{D} 1\right\|_{\infty}^{-1} e^{-\alpha d^{\prime}} t\right) .
$$


Take $r^{\prime}>0$ such that $r^{\prime} \in\left(d^{\prime}, d^{\prime}+\varepsilon\right)$ for any $\varepsilon>0$. Applying $t=e^{\alpha r^{\prime}}$ to (12), we see then

$$
\begin{aligned}
\mathbf{P}_{m_{\alpha}}^{\alpha w, *}\left(e^{\alpha r^{\prime}}<\tau_{D}^{\alpha w}\right) & =\mathbf{P}_{m_{\alpha}}^{\alpha w, D}\left(e^{\alpha r^{\prime}}<\tau_{D}^{\alpha w}\right) \\
& =Z_{\alpha}^{-1} \int_{D} u_{\alpha}^{D}\left(e^{\alpha r^{\prime}}, x\right) e^{-\alpha w(x)} d x \\
& \leq e^{\alpha\left(\inf _{D} w+\varepsilon\right)} H_{\alpha}^{D}\left(e^{\alpha r^{\prime}}\right)^{1 / 2} \longrightarrow 0 \quad \text { as } \alpha \rightarrow \infty
\end{aligned}
$$

which implies that

$$
\int_{D} h_{e^{-\alpha\left(d^{\prime}+\varepsilon\right)}}^{\alpha w} d m_{\alpha} \longrightarrow 1 \quad \text { as } \alpha \rightarrow \infty .
$$

Now, (i) of the lemma is a consequence of (9) and (13).

The first assertion of (ii) was proved in Theorem [6] (also, in Theorem II.1 in [8] related to the reflected Dirichlet form $\left(\mathcal{E}^{\alpha w, D}, H^{1}(D)\right)$ under the smoothness of $D$ ). Finally, we prove the last assertion of (ii). The idea of the proof is originally due to [8], but we need to improve some technical tools in our settings ( $D$ is not smooth). Let $B$ be an open ball such that $\bar{B} \subset D \equiv D_{r}(w)(r>0)$ and $r_{B}:=\max _{\bar{B}} w \in(0, d)$. Let $B_{1}$ be an open ball such that $B_{1} \subset B$ and $r_{B_{1}}=\max _{\bar{B}_{1}} w \in(0, d-c)$. Then for any $\varepsilon \in\left(0, d-c-r_{B_{1}}\right)$,

$$
\begin{aligned}
& \left\|h_{k \theta(\alpha)}^{\alpha w}-\int_{D} h_{k \theta(\alpha)}^{\alpha w} d m_{\alpha}\right\|_{L^{2}\left(B_{1}\right)}^{2} \\
\leq & e^{\alpha r_{B_{1}}}\left\|h_{k \theta(\alpha)}^{\alpha w}-\int_{D} h_{k \theta(\alpha)}^{\alpha w} d m_{\alpha}\right\|_{L^{2}\left(B_{1} ; e^{-\alpha w} d x\right)}^{2} \\
\leq & \gamma(\alpha)^{-1} e^{\alpha r_{B_{1}} \mathcal{E}_{k \theta(\alpha)}^{\alpha w, D}}\left(h_{k \theta(\alpha)}^{\alpha w}, h_{k \theta(\alpha)}^{\alpha w}\right) \\
\leq & \prec-\left(d-c-r_{B_{1}}-\varepsilon\right)
\end{aligned}
$$

by virtue of Lemma 2.3, Lemma 3.1(i) and the fact that for $\beta>0$

$$
\mathcal{E}_{\beta}^{\alpha w, D}\left(h_{\beta}^{\alpha w}, h_{\beta}^{\alpha w}\right)=\mathcal{E}_{\beta}^{\alpha w, D}\left(h_{\beta}^{\alpha w}, 1\right)=\beta \int_{D} h_{\beta}^{\alpha w} e^{-\alpha w} d x \leq \beta .
$$

Therefore we see that (8) holds for the open ball $B_{1}$ by combining (14) and the first assertion of (ii). On the other hand, by Poincaré inequality related to $H^{1}(B)$ for the open ball $B$, there exists a constant $\gamma_{B}^{-1}$ such that for any $\varepsilon \in\left(0, d-r_{B}\right)$,

$$
\begin{aligned}
\left\|h_{k \theta(\alpha)}^{\alpha w}-\left\langle h_{k \theta(\alpha)}^{\alpha w}\right\rangle_{B}\right\|_{L^{2}(B)}^{2} & \leq \gamma_{B}^{-1} \int_{B}\left|\nabla h_{k \theta(\alpha)}^{\alpha w}\right|^{2} d x \\
& \leq 2 \gamma_{B}^{-1} e^{\alpha r_{B}} \mathcal{E}_{k \theta(\alpha)}^{\alpha w, D}\left(h_{k \theta(\alpha)}^{\alpha w}, h_{k \theta(\alpha)}^{\alpha w}\right) \\
& \prec-\left(d-r_{B}-\varepsilon\right),
\end{aligned}
$$


where $\langle h\rangle_{B}:=\int_{B} h d x / \int_{B} d x$. Hence, for the open balls $B_{1}$ and $B$,

$$
\left\|h_{k \theta(\alpha)}^{\alpha w}-\frac{1}{1+k}\right\|_{L^{2}\left(B_{1}\right)} \longrightarrow 0, \quad\left\|h_{k \theta(\alpha)}^{\alpha w}-\left\langle h_{k \theta(\alpha)}^{\alpha w}\right\rangle_{B}\right\|_{L^{2}\left(B_{1}\right)} \longrightarrow 0
$$

as $\alpha \rightarrow \infty$ and thus

$$
\left\langle h_{k \theta(\alpha)}^{\alpha w}\right\rangle_{B} \rightarrow \frac{1}{1+k} \quad \text { as } \alpha \rightarrow \infty .
$$

Now, applying (16) to (15), we conclude that (8) also holds for any open ball $B$ such that $\bar{B} \subset D$.

Lemma 3.2. Let $f$ be a probability density function on $D \equiv D_{r}(w)(r>0)$ and let $f_{\alpha}$ be the scaled function of $f$ defined in (4). Then for any $k>0$,

$$
\int_{D} h_{k \theta(\alpha)}^{\alpha w} d f_{\alpha} \longrightarrow \frac{1}{1+k} \quad \text { as } \alpha \rightarrow \infty \text {. }
$$

Proof. Let $B_{0}$ be an open ball centered at 0 satisfying $\bar{B}_{0} \subset D$. In view of the proof of the last assertion of Lemma 3.1(ii), we see that (8) holds for $B_{0}$ and its speed of decay is exponential. Note that the support of $f_{\alpha}$ is contained in $B_{0}$ for large enough $\alpha$. So by the similar argument in the proof of Theorem II.3 in [8], we have

$$
\begin{aligned}
& \left\|h_{k \theta(\alpha)}^{\alpha w}-\frac{1}{1+k}\right\|_{L^{2}\left(D ; f_{\alpha} d x\right)} \\
\leq & 4 \int_{\{f \geq N\}} f(x) d x+\alpha^{\lambda n} N\left\|h_{k \theta(\alpha)}^{\alpha w}-\frac{1}{1+k}\right\|_{L^{2}\left(B_{0}\right)} \longrightarrow 0
\end{aligned}
$$

by letting $\alpha \rightarrow \infty$ and $N \rightarrow \infty$. This ends the proof of the lemma.

\section{Proof of Theorem 1.1}

In what follows, let $d_{H}$ be the Hausdorff distance on a family of non-empty compact sets $\mathcal{K}$ of $\mathbf{R}^{n}$. Clearly, $\overline{D_{r}(w)}$ (for fixed $w$ ) is a non-decreasing set valued function and is an element of $\mathcal{K}$ for any $r>0$.

Lemma 4.1. For Q-a.s., $\overline{D .(w)}$ is continuous on $(0, \infty)$ with respect to $d_{H}$.

Proof. First, we prove that (for fixed $w) \overline{D .(w)}$ is left continuous on $(0, \infty)$ with respect to $d_{H}$. To do this, it suffices to show that for any $\varepsilon>0$, there exists $s \in(0, r)$ such that $U_{\varepsilon}\left(\overline{D_{s}(w)}\right) \supset \overline{D_{r}(w)}$. Set

$$
\ell_{s}(x):=\inf _{y \in \overline{D_{s}(w)}}|x-y|, \quad x \in \overline{D_{r}(w)} .
$$

Then $\ell_{s}(\cdot)$ is a continuous function on $\overline{D_{r}(w)}$. Moreover, $\lim _{s \uparrow r} \ell_{s}(x)=0$ for any $x \in \overline{D_{r}(w)}$. Indeed, by the connectedness of $D_{r}(w)$, there exists a continuous path $\phi:[0,1] \rightarrow D_{r}(w)$ such that $\phi(0)=0$ and $\phi(1)=x$ for $x \in D_{r}(w)$. For this $\phi$, we can choose $s>0$ such that $s \in\left(\sup _{t \in[0,1]} w(\phi(t)), r\right)$ 
and $x \in D_{s}(w)$. Therefore we see that $\ell_{s}(x)$ converges uniformly to 0 on $\overline{D_{r}(w)}$ as $s \uparrow r$ and consequently, there exists $s \in(0, r)$ such that $\ell_{s}(x)<\varepsilon(\varepsilon>0)$ for all $x \in \overline{D_{r}(w)}$. Now, we prove the assertion of the lemma. Let $J(w)$ be the set of discontinuous points of $\overline{D_{r}(w)}$. By the left continuity of $\overline{D .(w)}, J(w)$ is a denumerable set. Therefore $\int_{J(w)} d r=0$ and

$$
\int_{0}^{\infty} Q(r \in J(w)) d r=\mathbf{E}^{Q}\left(\int_{J(w)} d r\right)=0
$$

where $\mathbf{E}^{Q}$ denotes the expectation related to $(\mathcal{W}, Q)$. Since for any $\alpha>0$ and $r>0$

$$
D_{r}\left(w_{\alpha}\right)=\alpha^{-\lambda} D_{\alpha r}(w),
$$

the $\lambda^{-1}$-self-similarity of the environment implies that

$$
Q(r \in J(w))=Q\left(r \in J\left(w_{\alpha}\right)\right)=Q(\alpha r \in J(w))=0 .
$$

Hence we see that $Q(r \in J(w))=0$ does not depend on $r>0$ and we obtain the desired result.

Lemma 4.2. Let $f, f_{\alpha}$ and $D$ be the same as in Lemma 3.2. Let $r(\alpha)$ be a function such that $r(\alpha) \rightarrow r(r>0)$ as $\alpha \rightarrow \infty$. Then

$$
\left|\mathbf{P}_{f_{\alpha}}^{\alpha w, *}\left(X\left(e^{\alpha r(\alpha)}\right) \in B\right)-m_{\alpha}(B)\right| \longrightarrow 0 \quad \text { as } \alpha \rightarrow \infty
$$

for any open ball $B$ such that $\bar{B} \subset D$.

Proof. Note that it makes no difference to replace an environment $w$ by $w-$ $\inf _{D} w$ as far as the critical depth $c$, diffusion $\left\{X(t), \mathbf{P}_{x}^{\alpha w, *}, x \in D^{*}\right\}$ and the associated spectral gap $\gamma(\alpha)$ are considered. So we may assume $\inf _{D} w=0$ without loss of generality. Let $\left\{p_{t}^{\alpha w, *}\right\}_{t>0}$ be the $L^{2}\left(D ; m_{\alpha}\right)$-semigroup of $\left\{X(t), \mathbf{P}_{x}^{\alpha w, *}, x \in D^{*}\right\}$ and $\left\{F_{\gamma}^{\alpha w, *}\right\}$ the associated spectral family of $\left\{p_{t}^{\alpha w, *}\right\}_{t>0}$, that is, $p_{t}^{\alpha w, *}=\int_{0}^{\infty} e^{-\gamma t} d F_{\gamma}^{\alpha w, *}$. Take a large enough $\alpha>0$ satisfying $c<r(\alpha)$. For an open ball $B$ such that $\bar{B} \subset D$, define the function $g$ on $D$ by $g(x)=I_{B}(x)-m_{\alpha}(B)$. Then

$$
\begin{aligned}
& \left|\mathbf{P}_{f_{\alpha}}^{\alpha w, *}\left(X\left(e^{\alpha r(\alpha)}\right) \in B\right)-m_{\alpha}(B)\right| \\
= & \int_{D}\left|\mathbf{E}_{x}^{\alpha w, *}\left(g\left(X\left(e^{\alpha r(\alpha)}\right)\right)\right)\right| f_{\alpha}(x) d x \\
\leq & \int_{\{f \geq N\}} f(x) d x+\alpha^{\lambda n} N \int_{D}\left|p_{e^{\alpha r(\alpha)}}^{\alpha w, *} g(x)\right| d x \\
\leq & \int_{\{f \geq N\}} f(x) d x+\alpha^{\lambda n} N Z_{\alpha} e^{\alpha r}\left\|p_{e^{\alpha r(\alpha)}}^{\alpha w, *} g\right\|_{L^{2}\left(D ; m_{\alpha}\right)} .
\end{aligned}
$$


On the other hand, by Lemma 2.3 and the fact that $\|g\|_{L^{1}\left(D ; m_{\alpha}\right)}=0$,

$$
\begin{aligned}
\left\|p_{e^{\alpha r(\alpha)}}^{\alpha w, *} g\right\|_{L^{2}\left(D ; m_{\alpha}\right)}^{2} & =\int_{\gamma(\alpha)}^{\infty} \exp \left(-2 \gamma e^{\alpha r(\alpha)}\right) d\left(F_{\gamma}^{\alpha w, *} g, g\right)_{L^{2}\left(D ; m_{\alpha}\right)} \\
& \leq \exp \left(-2 \gamma(\alpha) e^{\alpha r(\alpha)}\|g\|_{L^{2}\left(D ; m_{\alpha}\right)}^{2}\right. \\
& \leq \exp \left(-2 e^{\alpha(r(\alpha)-c-\varepsilon)}\right)
\end{aligned}
$$

for any $\varepsilon \in(0, r(\alpha)-c)$. Applying this relation to (19), we obtain the lemma by letting $\alpha \rightarrow \infty$ and $N \rightarrow \infty$.

Now, we are prepared to prove our main theorem.

Proof of Theorem 1.1. In view of (7) and its related remark mentioned right after, it holds that

$$
\left\{\alpha^{-2} \mathcal{X}\left(e^{\alpha r}\right), \mathcal{P}_{f}\right\} \stackrel{d}{=}\left\{\mathcal{X}\left(e^{\alpha r(\alpha)}\right), \mathcal{P}_{f_{\alpha}}^{\alpha}\right\}
$$

where $r(\alpha)=r-(2 \lambda / \alpha) \log \alpha, \mathcal{P}_{x}^{\alpha}(d w d \omega)=Q(d w) \mathbf{P}_{x}^{\alpha w}(d \omega)$ and $f_{\alpha}$ is the scaled function of $f$ defined in (4). Using this, we shall prove that for any $\varepsilon>0$

$$
\mathcal{P}_{f_{\alpha}}^{\alpha}\left(d_{H}\left(\mathcal{X}\left(e^{\alpha r(\alpha)}\right), \overline{D_{r}(w)}\right)>\varepsilon\right) \longrightarrow 0 \quad \text { as } \alpha \rightarrow \infty .
$$

First, take $\varepsilon^{\prime}$ such that $\varepsilon^{\prime} \in(0, r-r(\alpha))$ and apply $k=e^{\alpha \varepsilon^{\prime}}$ to (17). Then by Lemma 3.1(i), we have

$$
\begin{aligned}
\mathbf{P}_{f_{\alpha}}^{\alpha w}\left(\tau_{D_{r}(w)}^{\alpha w}<e^{\alpha r(\alpha)}\right) & =\mathbf{P}_{f_{\alpha}}^{\alpha w, *}\left(\tau_{D_{r}(w)}^{\alpha w}<e^{\alpha r(\alpha)}\right) \\
& \leq e \int_{D_{r}(w)} h_{e^{\alpha \varepsilon^{\prime}} e^{-\alpha\left(r(\alpha)+\varepsilon^{\prime}\right)}}^{\alpha w} f_{\alpha} d x \\
& \leq e \int_{D_{r}(w)} h_{e^{\alpha \varepsilon^{\prime} \theta(\alpha)}}^{\alpha w} f_{\alpha} d x \longrightarrow 0 \text { as } \alpha \rightarrow \infty
\end{aligned}
$$

which implies that for any $\varepsilon>0, \mathcal{X}\left(e^{\alpha r(\alpha)}\right) \subset U_{\varepsilon}\left(\overline{D_{r}(w)}\right), \mathcal{P}_{f_{\alpha}}^{\alpha}$-a.s. as $\alpha \rightarrow \infty$. Now, it remains to prove that under $\mathcal{P}_{f_{\alpha}}^{\alpha}$,

$$
\overline{D_{r}(w)} \subset U_{\varepsilon}\left(\mathcal{X}\left(e^{\alpha r(\alpha)}\right)\right) \quad \text { as } \alpha \rightarrow \infty .
$$

To this end, let $B(x)$ be an open ball of $D_{r}(w)$ centered at $x$ with radius $\varepsilon^{\prime \prime} / 2$, where $x \in \overline{D_{r-\varepsilon^{\prime \prime} / 2}(w)}$ and $\varepsilon^{\prime \prime} \in(0, \varepsilon)$. By the same reason in the proof of Lemma 4.2 , we may assume that $\inf _{D_{r}(w)} w=0$. Consider a modified environment $\widetilde{w}(x)$ of $w(x)$ on $\overline{D_{r}(w)}$ relative to $B(x)$ defined as follows:

$$
\widetilde{w}(x)=w(x) \text { on } B(x)^{c}, \quad \widetilde{w}(x) \leq w(x) \text { on } \overline{B(x)}
$$

and

$$
\inf _{x \in \overline{B(x)}} \widetilde{w}(x)=-\delta(\delta>0) .
$$


For this $\widetilde{w}$, let consider the Dirichlet form $\left(\mathcal{E}^{\alpha \widetilde{w}}, H_{*}^{1}\left(D_{r}(w)\right)\right)$, the associated diffusion $\left\{X(t), \mathbf{P}_{x}^{\alpha \widetilde{w}, *}, x \in D_{r}(w)^{*}\right\}$, the normalized underlying measure $\widetilde{m}_{\alpha}$ on $D_{r}(w)$, the depth $\widetilde{d}$ and the critical depth $\widetilde{c}$ of $D_{r}(w)$ :

$$
\widetilde{d}=d\left(D_{r}(w), \widetilde{w}\right), \quad \widetilde{c}=c\left(D_{r}(w), \widetilde{w}\right)=\sup _{x, y \in D_{r}(w)} c_{x, y}(\widetilde{w})
$$

with

$$
c_{x, y}(\widetilde{w})=\inf _{\phi} \sup _{t \in[0,1]} \widetilde{w}(\phi(t))-\widetilde{w}(x)-\widetilde{w}(y)-\delta,
$$

in a similar way of Section 2. Then since $D_{r}(w)$ is also a sub-level domain of $\widetilde{w}$, it is easy to check that $\widetilde{m}_{\alpha}(B(x)) \rightarrow 1$ as $\alpha \rightarrow \infty$ and

$$
\widetilde{c}<r+\delta
$$

In particular, we claim that $(21)$ can be regarded as $\widetilde{c}<r$ by choosing sufficiently small $\delta>0$. Indeed, let $\widetilde{E}\left(r_{0}\right)$ be a connected component of the level set $\left\{x \in D_{r}(w): \widetilde{w}(x)<r_{0}, \sup _{\overline{B(x)}} w<r_{0}<r\right\}$ containing $\overline{B(x)}$. Then,

$$
\widetilde{c}_{1}:=\sup _{x \in D_{r}(w) \backslash \widetilde{E}\left(r_{0}\right), y \in \widetilde{E}\left(r_{0}\right)} c_{x, y}(\widetilde{w}) \quad \text { and } \quad \widetilde{c}_{2}:=\sup _{x, y \in D_{r}(w) \backslash \widetilde{E}\left(r_{0}\right)} c_{x, y}(\widetilde{w})
$$

are strictly less than $r$ by the definition of the critical depth. On the other hand, since $\widetilde{E}\left(r_{0}\right)$ is also a sub-level domain of $\widetilde{w}$,

$$
\widetilde{c}_{3}:=\sup _{x, y \in \widetilde{E}\left(r_{0}\right)} c_{x, y}(\widetilde{w})=c\left(\widetilde{E}\left(r_{0}\right), \widetilde{w}\right)<d\left(\widetilde{E}\left(r_{0}\right), \widetilde{w}\right)=r_{0}+\delta
$$

and thus, $\widetilde{c}_{3}$ is also strictly less than $r$ by choosing the sufficiently small $\delta>0$. Noting $\widetilde{c}=\max \left\{\widetilde{c}_{1}, \widetilde{c}_{2}, \widetilde{c}_{3}\right\}$ we conclude that the claim is true. Therefore we see that $\widetilde{c}<r(\alpha)$ for sufficiently large $\alpha>0$ and

$$
\mathbf{P}_{f_{\alpha}}^{\alpha \widetilde{w}, *}\left(\sigma_{B(x)}^{\alpha \widetilde{w}}<e^{\alpha r(\alpha)}\right) \geq \mathbf{P}_{f_{\alpha}}^{\alpha \widetilde{w}, *}\left(X\left(e^{\alpha r(\alpha)}\right) \in B(x)\right) \longrightarrow 1 \quad \text { as } \alpha \rightarrow \infty
$$

by virtue of Lemma 4.2. Here $\sigma_{B}^{\alpha \widetilde{w}}$ denotes the first hitting time of the diffusion $X(t)$ to $B$. Since the processes $\left\{X(t), \mathbf{P}_{x}^{\alpha w}\right\}$ and $\left\{X(t), \mathbf{P}_{x}^{\alpha \widetilde{w}}\right\}$ have the same law on $B(x)^{c}$,

$$
\mathbf{P}_{f_{\alpha}}^{\alpha w}\left(\sigma_{B(x)}^{\alpha w}<e^{\alpha r(\alpha)}\right)=\mathbf{P}_{f_{\alpha}}^{\alpha \widetilde{w}, *}\left(\sigma_{B(x)}^{\alpha \widetilde{w}}<e^{\alpha r(\alpha)}\right) \longrightarrow 1 \quad \text { as } \alpha \rightarrow \infty
$$

which implies that $B(x) \subset U_{\varepsilon}\left(\mathcal{X}\left(e^{\alpha r(\alpha)}\right)\right), \mathbf{P}_{f_{\alpha}}^{\alpha w}$-a.s.. Hence we have

$$
\overline{D_{r-\varepsilon^{\prime \prime}}(w)} \subset U_{\varepsilon}\left(\mathcal{X}\left(e^{\alpha r(\alpha)}\right)\right) \quad \text { as } \alpha \rightarrow \infty
$$

under $\mathcal{P}_{f_{\alpha}}^{\alpha}$. Letting $\varepsilon^{\prime \prime} \rightarrow 0$, we can derive (20) from Lemma 4.1.

Remark 4.3. (i) Excepting the one dimensional case, we do not know how to determine the compact set $K(w, r) \subset \mathbf{R}^{n}$ such that under $\mathcal{P}_{f}, \alpha^{-\lambda} \mathcal{X}\left(e^{\alpha r}\right)$ converges in probability to $K(w, r)$ as $\alpha \rightarrow \infty$ without the second assumption of (A.2). 
(ii) It is possible to apply our result to an arbitrary initial distribution (that is, a point). To deduce this, one may use a priori Gaussian bounds on the transition probabilities.

Acknowledgement. A part of this paper is base on my talk in "International Workshop on Probability Theory and its Applications" held at Seoul National University, Korea. I am grateful to Professor Panki Kim for his kind invitation.

\section{References}

[1] T. Brox, A one-dimensional diffusion process in a Wiener medium, Ann. Probab. 14 (1986), no. 4, 1206-1218.

[2] M. Fukushima, Y. Oshima, and M. Takeda, Dirichlet Forms and Symmetric Markov Processes, de Gruyter Studies in Mathematics, 19. Walter de Gruyter \& Co., Berlin, 1994.

[3] M. Fukushima and H. Tanaka, Poisson point processes attached to symmetric diffusions, Ann. Inst. H. Poincaré Probab. Statist. 41 (2005), no. 3, 419-459.

[4] R. A. Holley, S. Kusuoka, and D. W. Stroock, Asymptotics of the spectral gap with applications to the theory of simulated annealing, J. Funct. Anal. 83 (1989), no. 2, $333-347$.

[5] K. Kawazu, Y. Tamura, and H. Tanaka, Limit theorems for one-dimensional diffusions and random walks in random environments, Probab. Theory Related Fields 80 (1989), no. $4,501-541$.

[6] D. Kim, On spectral gaps and exit time distributions for a non-smooth domain, Forum Math. 18 (2006), no. 4, 571-583.

[7] D. Kim and Y. Oshima, Some inequalities related to transience and recurrence of Markov processes and their applications, J. Theor. Probab. 23 (2010), no. 1, 148-168.

[8] P. Mathieu, Zero white noise limit through Dirichlet forms, with application to diffusions in a random medium, Probab. Theory Related Fields 99 (1994), no. 4, 549-580.

[9] Limit theorems for diffusions with a random potential, Stochastic Process. Appl. 60 (1995), no. 1, 103-111.

[10] Y. G. Sinai, The limit behavior of a one-dimensional random walk in a random environment, Teor. Veroyatnost. i Primenen. 27 (1982), no. 2, 247-258.

[11] P. Stollmann and J. Voigt, Perturbation of Dirichlet forms by measures, Potential Anal. 5 (1996), no. 2, 109-138.

[12] H. Takahashi, Recurrence and transience of multi-dimensional diffusion processes in reflected Brownian environments, Statist. Probab. Lett. 69 (2004), no. 2, 171-174.

[13] H. Tanaka, Recurrence of a diffusion process in a multidimensional Brownian environment, Proc. Japan Acad. Ser. A Math. Sci. 69 (1993), no. 9, 377-381.

Department of Mathematics and Engineering

FACULTY OF ENGINEERING

KuMAMOTO UNIVERSITY

KUMAMOTO 860-8555, JAPAN

E-mail address: daehong@gpo.kumamoto-u.ac.jp 